\title{
MACROALGAS MARINAS BENTÓNICAS DEL SUBMAREAL SOMERO DE LA ECORREGIÓN SUBANTÁRTICA DE MAGALLANES, CHILE
}

\author{
SHALLOW SUBTIDAL BENTHIC MARINE MACROALGAE FROM THE MAGELLAN \\ SUBANTARCTIC ECOREGION, CHILE
}

\author{
Andrés Mansilla ${ }^{1,4}$, Marcela Ávila ${ }^{2}$, María E. Ramírez ${ }^{3}$, Juan Pablo Rodriguez ${ }^{1,4}$, Sebastián \\ Rosenfeld ${ }^{1,4,5}$, Jaime Ojeda ${ }^{1}$, \& Johanna Marambio ${ }^{1,5}$
}

\begin{abstract}
The area of channels and fjords belonging to the Magellan subantarctic has a high diversity of macroalgae, in relation to the temperate areas of South America. Geomorphological changes, the geographical position in a high latitude together with the climate change in this region create a series of coast marine habitats with a significant environmental heterogeneity, where a diverse benthic marine flora take place, which are not still known, that has evolved in an adaptive way to these changes. This research was developed under the 16 fjords CIMAR program in 2010, aboard the scientific research vessel Abate Molina, under the "Marine Benthic macroalgae of the Region of Magallanes and Chilean Antarctica "project. This paper gives information about a total of 57 species of macroalgae in the region, with representatives of the Rhodophyta (51\%), Ochrophyta (Class Phaeophyceae) (37\%) and Divisions Chlorophyta (12\%). Takes into account the floristic affinities of the sectors studied and deals with the flora of the Antarctic continent, and also incorporates 7 novel macroalgal references to the subantarctic Magellan ecoregion.
\end{abstract}

Key word: Seaweed, floristic composition, distribution, Magallanes, Sub-Antarctic ecosystem.

\footnotetext{
${ }^{1}$ Laboratorio de Macroalgas Antárticas y Subantárticas, Departamento de Ciencias y Recursos Naturales, Facultad de Ciencias, Universidad de Magallanes.

${ }^{2}$ Instituto de Ciencia y Tecnología, Universidad Arturo Prat, Puerto Montt.

${ }^{3}$ Museo Nacional de Historia Natural, Área de Botánica, casilla 787, Santiago, Chile.

${ }^{4}$ Instituto de Ecología y Biodiversidad, IEB Chile.

${ }^{5}$ Magister en Ciencias Mención Manejo y Conservación en Recursos Naturales en Ambientes Subantárticos, Universidad de Magallanes.
} 


\section{RESUMEN}

La zona de canales y fiordos subantárticos de Magallanes, presenta una alta diversidad de macroalgas, respecto a las zonas templadas del sur de Sudamérica. Sus cambios geomorfológicos, su posicionamiento geográfico de alta latitud y sus cambios estacionales, crean en esta región una serie de hábitats marino-costeros con una importante heterogeneidad ambiental donde ocurre una diversa flora marina bentónica. Esta biota ha evolucionado de manera adaptativa a estos cambios y requiere ser investigada. El presente estudio fue desarrollado en el marco del programa CIMAR fiordos 16 realizado el año 2010 a bordo de la nave de investigación científica Abate Molina. Se realizaron muestreos mediante buceo autónomo sobre la diversidad de macroalgas, en 17 sitios de los canales y fiordos magallánicos. Un total de 57 especies de macroalgas fueron recolectadas e identificadas para la región, con representantes de las Divisiones Rhodophyta (51\%) Ochrophyta (Clase Phaeophyceae) (37\%) y Chlorophyta (12\%). Se abordan las afinidades florísticas de los sectores estudiados y con la flora del continente antártico. Se registran 7 nuevos taxa para la eco-región subantártica de Magallanes.

Palabras clave: Algas marinas, composición florística, distribución, Magallanes, ecosistemas subantárticos.

\section{INTRODUCCIÓN}

La región de Magallanes con una superficie de $132.033 \mathrm{~km}^{2}$ (Pérez, 2000), es el área representativa de los ambientes subantárticos de mayor extensión en el mundo (Rozzi et al. 2012). Desde un punto de vista biológico, es una provincia biogeográfica con alto grado de endemismo, pero que incluye también componentes antárticos y especies presentes en el centro-norte del continente sudamericano (Meneses \& Santelices, 2000). Por su difícil acceso debido a su topografía y clima poco amigable para la exploración, la región de Magallanes tiene un impacto relativo de la actividad antrópica. Este hecho motivó a la UNESCO a decretar la zona más austral de Magallanes como Reserva de la Biosfera Cabo de Hornos, por ser considerada un "hot spot" de biodiversidad de organismos criptógamos (Rozzi, 2005'; Rozzi et al. 2006; 2007).

Debido a su heterogeneidad ambiental Magallanes presenta no solo una gran diversidad de algas, sino también de algunos grupos de invertebrados $y$ vertebrados marinos con un elevado grado de endemismo (Moreno \& Jara, 1984; Fernández et al. 2000; Camus, 2001).

1 Rozzi, R. 2005. Biodiversità e benessere: il caso del Sud America. www.filosofia-ambientale.it.
Principal atención, por su diversidad, merecen la región intermareal y submareal somera, que son precisamente las más pobremente estudiadas. La historia de la exploración de la región se inicia aproximadamente con las expediciones de Charles Darwin y el Capitán Robert FitzRoy (1834), quienes fueron los primeros en emprender un estudio metódico y sistemático de la biota accequible para la época. Posteriormente una serie de expediciones europeas entre las que se destacan: Mission Scientifique du Cap Horn (18821883), Wissennschaftliche Ergebnisse SudpolarExpedition (1901-1903), Deuxieme Expedition Antarctique Francaise (1908-1910), Botanische Ergebnisse der Schewedischen Expedition Nach Patagonien und dem Feuerlange (1907-1909), Nereis Australis (Algae of the Southern Ocean) (1947) y Lund University Chile Expedition (19481949). Sin embargo, son escasos los trabajos que aportan al conocimiento taxonómico de las macroalgas de esta región. Entre las contribuciones más destacadas tenemos los trabajos de Hariot, (1889), Skottsberg (1907); Kylin \& Skottsberg (1919); Lemoine (1920) y Levring (1960).

A mediados del siglo XX surgen las primeras contribuciones ficológicas australes de científicos chilenos, en particular las realizadas por Alveal et al. (1982), Ávila et al. (1982), Romo et al. (1984) y Santelices \& Ojeda (1984) en Puerto Toro e Isla Navarino, orientadas al estudio 


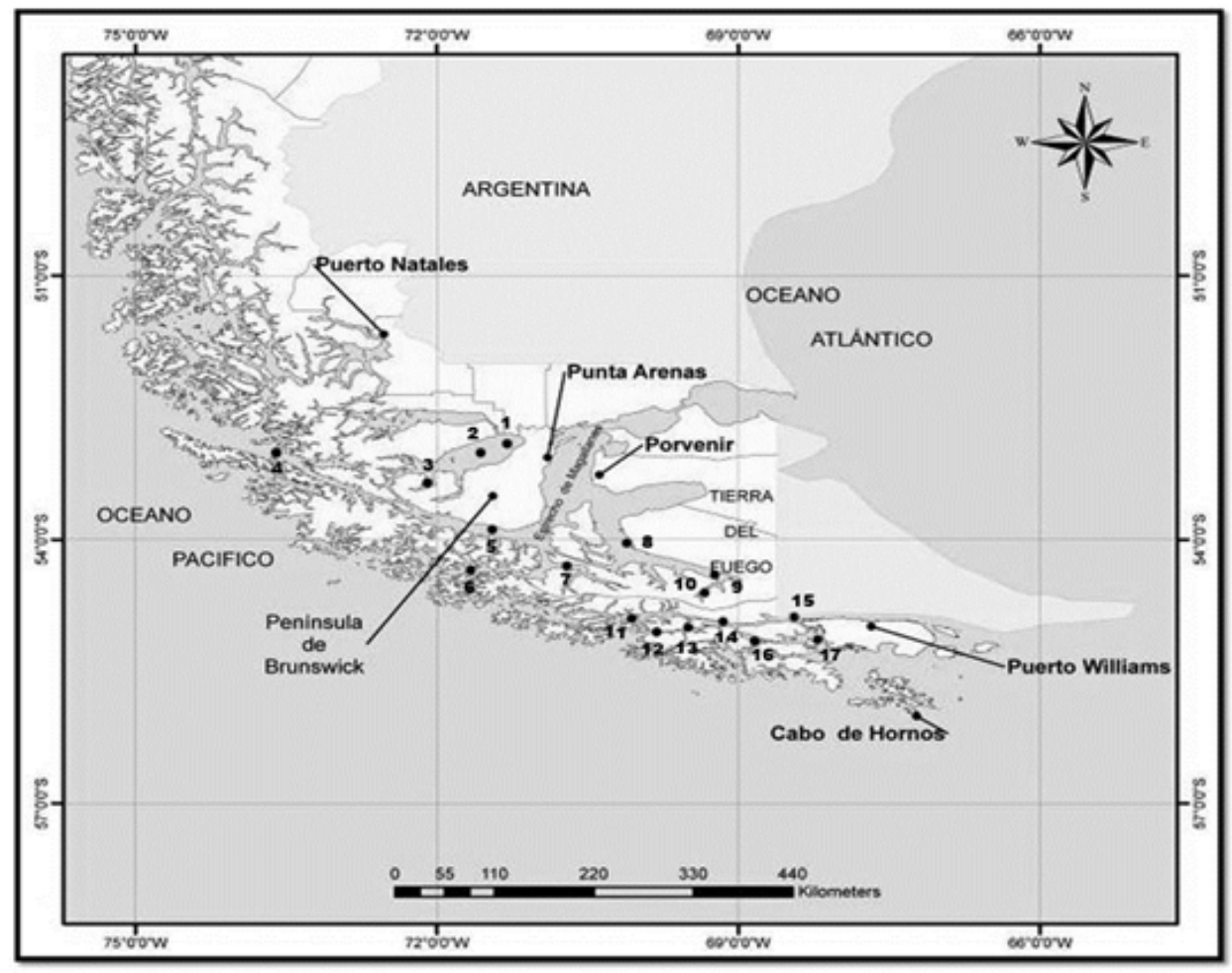

Fig. 1. Localización de las estaciones de muestreo en la región de Magallanes durante el programa CIMAR fiordos 16.

sobre la factibilidad de utilización de los bosques de Macrocystis pyrifera, donde se abordaron aspectos bio-ecológicos de estas poblaciones. Desde entonces, excepto algunas contribuciones (Ramírez \& Santelices, 1991; Ramírez, 1995, 2010; Mansilla \& Navarro, 2003), la flora marina de la región de Magallanes ha sido poco estudiada.

Existen aspectos de la flora marina bentónica que aún no han sido abordados, por ejemplo, escenarios biogeográficos de la macroflora de la región y sus conexiones con el continente antártico e islas subantárticas. A pesar de haberse descrito un elevado endemismo para la región, algunas de las especies tipo no han sido colectadas nuevamente y por lo tanto, no ha sido ratificada su distribución, existiendo un grado de incertidumbre respecto del número real de especies en la región. Por lo tanto, es urgente ratificar su presencia e identidad para conocer con exactitud el grado de endemismo en la región y para redescribir especies cuya determinación taxonómica ha sido errónea.

La región subantártica de Magallanes presenta un ecosistema marino caracterizado por presencia de glaciares, cambios de mareas, y corrientes, entre otros, que repercuten en la composición y distribución principalmente de los organismos bentónicos (Gerdes \& Montiel, 1999; Gutt et al. 1999; Ríos, 2007²; Ríos et al. 2010). La heterogeneidad ambiental existente en los ecosistemas marinos de la región de Magallanes se debe a los proceso de oscilaciones climáticas ocurridas durante el Cuaternario (Provan \& Bennett, 2008; Hewitt, 2000; 2004). En cuanto al continente antártico éste permaneció totalmente aislado hasta la apertura del paso Drake y el surgimiento de la corriente circumpolar antártica (CCA) ca. 38 millones de años AP (Barker, 2001;

2 Ríos, C. 2007. Marine benthic communities of the Magellan Region, Southern Chile: contribution of different habitats to the overall biodiversity. PhD Thesis, Alfred Wegener Institut for Polar and Marine Research-Bremen University, Germany. 122 pp. Disponible en http://nbn-resolving.de/urn:nbn:de:gbv:46diss000110638 
TABLA 1. Caracterización de las estaciones de colecta realizados en el marco del Programa CIMAR fiordos 16.

\begin{tabular}{|c|c|c|c|c|}
\hline Estación & Posición S & Posición W & Localización & Caracterización \\
\hline 1 & $52^{\circ} 59^{\prime} 15,7^{\prime \prime}$ & $71^{\circ} 14^{\prime} 37,1^{\prime \prime}$ & Seno Otway-Pinguineras & Playa de bolones de roca mediano, presencia de Huiro \\
\hline 2 & $52^{\circ} 56 ' 52,1^{\prime \prime}$ & $71^{\circ} 44^{\prime} 13^{\prime \prime}$ & Isla Riesco & Playa de bolones de roca mediano, presencia de Huiro \\
\hline 3 & $53^{\circ} 20^{\prime} 29,1^{\prime \prime}$ & $72^{\circ} 01^{\prime} 20,9^{\prime \prime}$ & Seno Otway-Entrada & $\begin{array}{l}\text { Playa de bolones de roca mediano con arena, presencia } \\
\text { de Huiro }\end{array}$ \\
\hline 4 & $53^{\circ} 11^{\prime} 30,7^{\prime \prime}$ & $73^{\circ} 23^{\prime} 17,4^{\prime \prime}$ & $\begin{array}{l}\text { Estrecho de Magallanes- Antes del } \\
\text { tortuoso }\end{array}$ & Playa de bolones de roca grande presencia de Huiro \\
\hline 5 & $53^{\circ} 56^{\prime} 29,6^{\prime \prime}$ & $71^{\circ} 34^{\prime} 46,8^{\prime \prime}$ & $\begin{array}{l}\text { Estrecho de Magallanes- Cabo } \\
\text { Holland }\end{array}$ & Playa de bolones de roca grande presencia de Huiro \\
\hline 6 & $54^{\circ} 19^{\prime} 21^{\prime \prime}$ & $71^{\circ} 38^{\prime} 22,1^{\prime \prime}$ & Canal Brecknock & Playa de bolones de roca grande presencia de Huiro \\
\hline 7 & $54^{\circ} 22^{\prime} 11,8^{\prime \prime}$ & $70^{\circ} 36^{\prime} 27,1^{\prime \prime}$ & Seno Agostini & Playa con arena y guijarros presencia de Huiro \\
\hline 8 & $54^{\circ} 24^{\prime} 20,9^{\prime \prime}$ & $69^{\circ} 35^{\prime} 52,6^{\prime \prime}$ & Entrada de Canal Parry Marinelli & Playa con arena y guijarros presencia de hielo \\
\hline 9 & $54^{\circ} 26^{\prime} 50,2^{\prime \prime}$ & $69^{\circ} 13^{\prime} 05,7^{\prime \prime}$ & Entrada de Canal Parry & Playa de bolones de roca grande presencia de Huiro \\
\hline 10 & $54^{\circ} 36^{\prime} 47,8^{\prime \prime}$ & $69^{\circ} 18^{\prime} 54,7^{\prime \prime}$ & Parry & playa de roca y arena con presencia de bosque de Huiro \\
\hline 11 & $54^{\circ} 57^{\prime} 31,1^{\prime \prime}$ & $70^{\circ} 44^{\prime} 06,9^{\prime \prime}$ & Final de Canal Ballenero & Playa de bolones de roca grande presencia de Huiro \\
\hline 12 & $55^{\circ} 04^{\prime} 18,4^{\prime \prime}$ & $69^{\circ} 48^{\prime} 59,1^{\prime \prime}$ & Brazo sur oeste Canal Beagle & Playa de bolones de roca grande presencia de Huiro \\
\hline 13 & $55^{\circ} 00^{\prime} 59,8^{\prime \prime}$ & $69^{\circ} 22^{\prime} 55,7^{\prime \prime}$ & Brazo sur oeste Canal Beagle & Playa de bolones de roca grande presencia de Huiro \\
\hline $14-15$ & $54^{\circ} 50^{\prime} 51,4^{\prime \prime}$ & $68^{\circ} 45^{\prime} 06,2^{\prime \prime}$ & Bahía Yendegahia & Playa con arena y guijarros presencia de Huiro \\
\hline 16 & $55^{\circ} 08^{\prime} 39,9^{\prime \prime}$ & $68^{\circ} 49^{\prime} 35^{\prime \prime}$ & Seno Ponsomby & Playa de bolones de roca grande presencia de Huiro \\
\hline 17 & $55^{\circ} 09^{\prime} 05,7^{\prime \prime}$ & $68^{\circ} 08^{\prime} 29,5^{\prime \prime}$ & Bahía Douglas & Playa de bolones de roca grande presencia de Huiro \\
\hline
\end{tabular}

Herraiz, 2009). Actualmente, es posible encontrar evidencias de estas conexiones a través de la flora y fauna existente entre Magallanes y Antártica (Arntz, 2005; Ramírez, 2010; Gonzalez-Wevar et al. 2010).

El objetivo de este trabajo es conocer la diversidad ficológica en las áreas de muestreo definidas en el Expedición CIMAR 16 FIORDOS realizada durante la primavera del 2010, entre las latitudes $52^{\circ} 56^{\prime} 52,1^{\prime \prime} \mathrm{S}$; 5509'05,7 "S y las longitudes $68^{\circ} 08^{\prime} 29,5^{\prime \prime} \mathrm{W}-73^{\circ} 23^{\prime} 17,4^{\prime \prime} \mathrm{W}$.

\section{MATERIAL Y MÉTODOS}

Estaciones de colecta

Las áreas de muestreo corresponden a las definidas en el Proyecto CIMAR 16 FIORDOS y están comprendidas entre las latitudes


680' $29,5^{\prime \prime}$ W - 73²3'17,4" W (Fig. 1 y Tabla
$1)$.

Las muestras de macroalgas fueron colectadas con ayuda del buque de investigación científica Abate Molina, en el período comprendido entre el 12 de octubre y el 11 de noviembre de 2010. Se realizaron 17 estaciones de muestreo de tipo submareal, las cuales por lo general estaban asociadas a bosques de Macrocystis pyrifera (Tabla 1). Las muestras submareales se recolectaron mediante buceo autónomo, realizando al menos 4 transectos verticales desde zonas someras hacia aguas profundas para cada estación. Una vez recolectadas las muestras, fueron separadas por divisiones (Chlorophyta, Ochrophyta (Clase Phaeophyceae) y Rhodophyta y posteriormente fijadas en formalina diluida en agua de mar al $4 \%$ y conservadas en bolsas plásticas debidamente etiquetadas para su posterior determinación taxonómica.

La determinación taxonómica de las 




Fig.2. Porcentaje de composición florística según las divisiones de macroalgas identificadas en el programa CIMAR FIORDOS 16.

macroalgas, se realizó mediante caracterizaciones y descripciones morfológicas macroscópicas y microscópicas, utilizando para ello microscopio estereoscópico marca Olympus, modelo SZ61 y microscopio óptico marca Olympus, modelo CX31. La identificación taxonómica fue realizada basándose en literatura especializada existente para otras regiones (Hoffmann \& Santelices, 1997; Mendoza \& Nizovoy, 2000; Wiencke \& Clayton, 2002; Boraso et al. 2003; Brodie et al. 2007). Luego de su determinación, las muestras fueron conservadas en seco en hojas de herbario, según la metodología indicada por Ramírez (1995). Además, se conservó un espécimen de cada especie en formalina al 4\%. Las exsicatas se encuentran depositadas en el herbario criptogámico de la Universidad de Magallanes.

Análisis de la información

El análisis multivariado se realizó utilizando los datos de presencia y ausencia de macroalgas, en cada una de las estaciones de muestreos. El análisis de clasificación y ordenación consintió primeramente en la construcción de una matriz de similitud entre los sectores de muestreo utilizando el coeficiente de similitud de Bray-Curtis (Bray y Curtis, 1957). Luego, para la clasificación se realizó un análisis de agrupación UPGMA (Unweighted
Pairgroup Method Using Arithmetic Averages; Sneath y Sokal, 1973), a partir del cálculo del indice de similitud de Bray-Curtis, construyendo un dendograma (o cluster), en el cual las estaciones de muestreo aparecen dispuestas en grupos en función de su similitud media. Las diferencias significativas en los agrupamientos (clusters) generados se evaluaron a través de la prueba SIMPROF $(\mathrm{P}<0,05$; Clarke et al., 2008).

Estos multivariados de biodiversidad se realizaron utilizando el programa PRIMER 6.0 (Clarke \& Gorley, 2005).

\section{RESULTADOS}

En el presente estudio se identificaron un total de 57 especies de macroalgas correspondientes a las divisiones Chlorophyta (12\%), Ochrophyta (Clase Phaeophyceae) (37\%) y Rhodophyta (51\%) (Fig. 2).

De la división Chlorophyta las especies más frecuentes fueron Codium fragile (Suringar) Hariot presente en tres estaciones de colecta $y$ Acrosiphonia pacifica (Montagne) J. Ag, presente en dos estaciones de colecta. Las especies menos frecuentes fueron Codium dimorphum Svedelius, Codium subantarcticum Silva sp. Ined, Ulothrix flacca (Dillwyn) Thuret, Ulva linza Linnaeus y Ulva prolifera (O.F. Müller) todas presentes en al menos 
TABLA 2. Ocurrencia de especies de las divisiones Chlorophyta, Ochrophyta (Clase Phaeophyceae) y Rhodophytaen las distintas estaciones muestreadas durante el programa CIMAR Fiordos 16. F= Frecuencia.

\begin{tabular}{llllllllllllllllll}
\hline Especie & & 1 & 2 & 3 & 4 & 5 & 6 & 7 & 8 & $9-10$ & 11 & 12 & 13 & $14-15$ & 16 & 17 & $\mathrm{~F}$
\end{tabular}

Division Chlorophyta

Acrosiphonia pacifica (Montagne) J. Ag. ***

Codium fragile (Suringar) Hariot

Codium dimorphum Svedelius

Codium subantarcticum Silva sp. Ined

Ulothrix flacca (Dillwyn) Thuret ****

Ulva linza Linnaeus

Ulva prolifera (O.F.Müller)***

Division Ochrophyta (Clase Phaeophyceae)

Adenocystis utricularis (Bory de Saint-Vincent) Skottsberg

Caepidium antarcticum J.Agardh

Chordaria magellanica Kylin

Cladostephus spongiosus (Hudson) C. Agardh

Cladothele decaisnei J.D.Hooker \& Harvey

Colpomenia sinuosa (Mertens ex Roth) Derbès \& Solier in

Castagne ${ }^{* * *}$

Desmarestia chordalis J.D. Hooker \& Harvey

Desmarestia confervoides (Bory de Saint-Vincent) M.E Ramirez \&

A.F.Peters

Desmarestia distans (C. Agardh) J. Agardh

Desmarestia ligulata (Stackhouse) J.V.Lamouroux

Desmarestia muellerii M.E. Ramirez \& A.F.Peters

Dictyota dichotoma (Hudson) J.V. Lamouroux ***

Durvillaea antarctica (Chamisso) Hariot

Ectocarpus siliculosus (Dillwyn) Lyngbye

Halopteris obovata (J.D.Hooker \& Harvey) Sauvageau ${ }^{* * * *}$

Lessonia flavicans Bory de Saint-Vincent in Dumont d'Urville

Lessonia $\mathrm{sp}$ *

Lessonia vadosa Searles

Macrocystis pyrifera (Linnaeus) C.Agardh

Scytosiphon lomentaria (Lyngbye) Link ****

$\begin{array}{llllllllllllllll}0 & 0 & 0 & 0 & 1 & 0 & 0 & 0 & 0 & 0 & 0 & 0 & 0 & 1 & 0 & 2 \\ 1 & 1 & 0 & 1 & 0 & 0 & 0 & 0 & 0 & 0 & 0 & 0 & 0 & 0 & 0 & 3 \\ 0 & 1 & 0 & 0 & 0 & 0 & 0 & 0 & 0 & 0 & 0 & 0 & 0 & 0 & 0 & 1 \\ 0 & 0 & 0 & 0 & 0 & 0 & 0 & 0 & 0 & 0 & 0 & 0 & 0 & 1 & 0 & 1 \\ 0 & 0 & 0 & 0 & 0 & 0 & 0 & 1 & 0 & 0 & 0 & 0 & 0 & 0 & 0 & 1 \\ 0 & 0 & 0 & 0 & 0 & 0 & 0 & 1 & 0 & 0 & 0 & 0 & 0 & 0 & 0 & 1 \\ 0 & 0 & 0 & 0 & 0 & 0 & 0 & 0 & 0 & 0 & 0 & 1 & 0 & 0 & 0 & 1\end{array}$

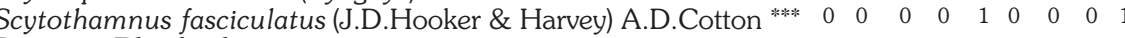

\section{Division Rhodophyta}

Acanthococcus antarcticus Hooker \& Harvey

Ahnfeltia plicata (Hudson) E.M.Fries ****

Ballia callitricha (C.Agardh) Kützing **

Bostrychia scorpioides (Hudson Montagne)

Callophyllis atrosanguinea (J.D. Hooker \& Harvey) Hariot ***:

Callophyllis variegata (Bory de Saint-Vincent) Kützing

Callymenia chilensis Levring *

Camontagnea oxyclada (Montagne) Pujals

Catenella fusiformis (J.Agardh) Skottsberg

Ceramium diaphanum (Lightfoot) Roth

Ceramium dozei Hariot

Ceramium stichidiosum J.Agardh

Ceramium virgatum Roth

Chondria secundata (J.Agardh) De Toni

Corallina officinalis flabellifera Schiffner

Delesseria fueguensis Skottsberg

Gigartina skottsbergii Setchell \& N.L.Gardner ****

Griffithsia antarctica Hooker f. \& Harvey *****

Heterosiphonia berkeleyi Montagne

Heterosiphonia merenia Falkenberg**

Iridaea cordata (Turner) Bory de Saint-Vincent ****

Leptofauchea chiloensis (Dalen \& G.W.Saunders)***

Lophurella hookeriana (J.Agardh) Falkenberg

Rhodymenia subantarctica R.W.Ricker

Mazzaella laminarioides (Bory) Fredericq

Nothogenia fastigiata (Bory de Saint-Vincent) P.G.Parkinson

Polysiphonia anisogona J.D.Hooker \& Harvey

Polysiphonia morrowii Harvey

$\begin{array}{lllllllll}1 & 1 & 0 & 1 & 1 & 1 & 0 & 1 & 1\end{array}$

$\begin{array}{lllllllll}0 & 0 & 1 & 1 & 1 & 1 & 0 & 0 & 1\end{array}$

$\begin{array}{lllllllll}0 & 0 & 0 & 0 & 0 & 0 & 0 & 0 & 1\end{array}$

$\begin{array}{lllllllll}1 & 0 & 0 & 0 & 0 & 0 & 0 & 0 & 0\end{array}$

$\begin{array}{lllllllll}0 & 0 & 0 & 0 & 0 & 0 & 0 & 0 & 0\end{array}$

$\begin{array}{lllllllll}0 & 0 & 0 & 0 & 1 & 0 & 0 & 0 & 0\end{array}$

$\begin{array}{lllllllll}0 & 0 & 0 & 0 & 0 & 1 & 0 & 0 & 0\end{array}$

$\begin{array}{lllllllll}1 & 0 & 0 & 1 & 1 & 0 & 1 & 0 & 0 \\ 0 & 0 & 0 & 0 & 1 & 1 & 0 & 0 & 0\end{array}$

$\begin{array}{lllllllll}0 & 0 & 0 & 0 & 1 & 1 & 0 & 0 & 0 \\ 0 & 0 & 0 & 0 & 0 & 1 & 0 & 0 & 0\end{array}$

$\begin{array}{lllllllll}0 & 0 & 0 & 0 & 0 & 1 & 0 & 0 & 0\end{array}$

$\begin{array}{lllllllll}0 & 0 & 0 & 0 & 1 & 0 & 0 & 0 & 0\end{array}$

$\begin{array}{lllllllll}0 & 0 & 0 & 1 & 1 & 1 & 0 & 0 & 1\end{array}$

$\begin{array}{lllllllll}0 & 0 & 0 & 0 & 1 & 0 & 0 & 0 & 0\end{array}$

$\begin{array}{lllllllll}1 & 0 & 0 & 1 & 0 & 1 & 0 & 0 & 0\end{array}$

$\begin{array}{lllllllll}0 & 0 & 0 & 1 & 1 & 1 & 0 & 0 & 1\end{array}$

$\begin{array}{lllllllll}0 & 0 & 0 & 0 & 0 & 0 & 0 & 0 & 0\end{array}$

$\begin{array}{lllllllll}0 & 0 & 0 & 1 & 1 & 1 & 0 & 0 & 0\end{array}$

$\begin{array}{lllllllll}1 & 1 & 1 & 1 & 1 & 1 & 1 & 1 & 1\end{array}$

$\begin{array}{lllllllll}0 & 0 & 1 & 0 & 0 & 0 & 1 & 1 & 0 \\ 0 & 0 & 0 & 0 & 1 & 0 & 0 & 0 & 1\end{array}$

$\begin{array}{lllllllll}1 & 1 & 0 & 1 & 0 & 0 & 0 & 0 & 0\end{array}$

$\begin{array}{lllllllll}1 & 0 & 0 & 0 & 0 & 0 & 0 & 0 & 1\end{array}$

$\begin{array}{lllllllll}1 & 1 & 0 & 1 & 1 & 1 & 0 & 0 & 0\end{array}$

$\begin{array}{lllllllll}0 & 0 & 0 & 0 & 0 & 0 & 0 & 0 & 0\end{array}$

$\begin{array}{lllllllll}0 & 0 & 0 & 1 & 0 & 0 & 0 & 0 & 0\end{array}$

$\begin{array}{lllllllll}0 & 0 & 0 & 1 & 0 & 0 & 0 & 0 & 0\end{array}$

$\begin{array}{lllllllll}0 & 0 & 0 & 0 & 0 & 0 & 0 & 0 & 0 \\ 0 & 0 & 0 & 0 & 1 & 0 & 0 & 0 & 0\end{array}$

$\begin{array}{lllllllll}0 & 0 & 0 & 0 & 1 & 0 & 0 & 0 & 0\end{array}$

$\begin{array}{lllllllll}0 & 0 & 0 & 0 & 0 & 0 & 0 & 0 & 0\end{array}$

$\begin{array}{lllllllll}1 & 0 & 0 & 0 & 0 & 0 & 0 & 0 & 0\end{array}$

$\begin{array}{lllllllll}1 & 0 & 0 & 0 & 0 & 0 & 0 & 0 & 0\end{array}$

$\begin{array}{lllllllll}1 & 0 & 0 & 0 & 0 & 0 & 0 & 0 & 0\end{array}$

$\begin{array}{lllllllll}1 & 0 & 0 & 0 & 1 & 0 & 0 & 0 & 0\end{array}$

$\begin{array}{lllllllll}1 & 0 & 0 & 1 & 0 & 0 & 0 & 0 & 0\end{array}$

$\begin{array}{lllllllll}0 & 0 & 0 & 0 & 0 & 1 & 0 & 0 & 0\end{array}$

$\begin{array}{lllllllll}0 & 0 & 0 & 0 & 0 & 0 & 0 & 0 & 0\end{array}$

$\begin{array}{lllllllll}0 & 1 & 1 & 1 & 1 & 0 & 1 & 0 & 0\end{array}$

$\begin{array}{lllllllll}0 & 0 & 0 & 0 & 0 & 0 & 0 & 0 & 0\end{array}$

$\begin{array}{lllllllll}0 & 0 & 0 & 0 & 0 & 0 & 0 & 0 & 0\end{array}$

$\begin{array}{lllllllll}0 & 0 & 0 & 0 & 1 & 0 & 0 & 0 & 0\end{array}$

$\begin{array}{lllllllll}0 & 0 & 1 & 0 & 0 & 0 & 0 & 0 & 0\end{array}$

$\begin{array}{lllllllll}0 & 0 & 0 & 0 & 0 & 0 & 0 & 0 & 0\end{array}$

$\begin{array}{lllllllll}1 & 1 & 1 & 1 & 1 & 1 & 1 & 0 & 1\end{array}$

$\begin{array}{lllllllll}0 & 0 & 0 & 0 & 0 & 0 & 0 & 0 & 0\end{array}$

$\begin{array}{lllllllll}0 & 0 & 0 & 0 & 0 & 1 & 0 & 0 & 0\end{array}$

$\begin{array}{lllllllll}0 & 0 & 0 & 0 & 0 & 0 & 1 & 0 & 1\end{array}$

$\begin{array}{lllllllll}0 & 0 & 0 & 0 & 1 & 0 & 0 & 0 & 0\end{array}$

$\begin{array}{lllllll}1 & 1 & 1 & 1 & 1 & 1 & 13\end{array}$

$\begin{array}{llll}0 & 1 & 0 & 0\end{array}$

$\begin{array}{llll}0 & 0 & 0 & 0\end{array}$

$\begin{array}{llll}0 & 0 & 0 & 0\end{array}$

$\begin{array}{lll}0 & 1 & 7\end{array}$

$\begin{array}{lll}0 & 0 & 1\end{array}$

$\begin{array}{lll}0 & 0 & 1\end{array}$

$\begin{array}{lllllll}1 & 0 & 1 & 0 & 0 & 0 & 2 \\ 0 & 0 & 0 & 0 & 0 & 1 & 2\end{array}$

Ptilonia magellanica (Montagne) J.Agardh

$\begin{array}{lllllllll}0 & 0 & 0 & 1 & 0 & 0 & 0 & 0 & 0\end{array}$

$\begin{array}{lllllll}0 & 0 & 0 & 0 & 0 & 0 & 1\end{array}$

Los símbolos indican"* Nueva referencia, en proceso de publicación de especie *** Nueva referencia para la Región de Magallanes, ${ }^{* * *}$ Especie presente en el continente Antártico. 


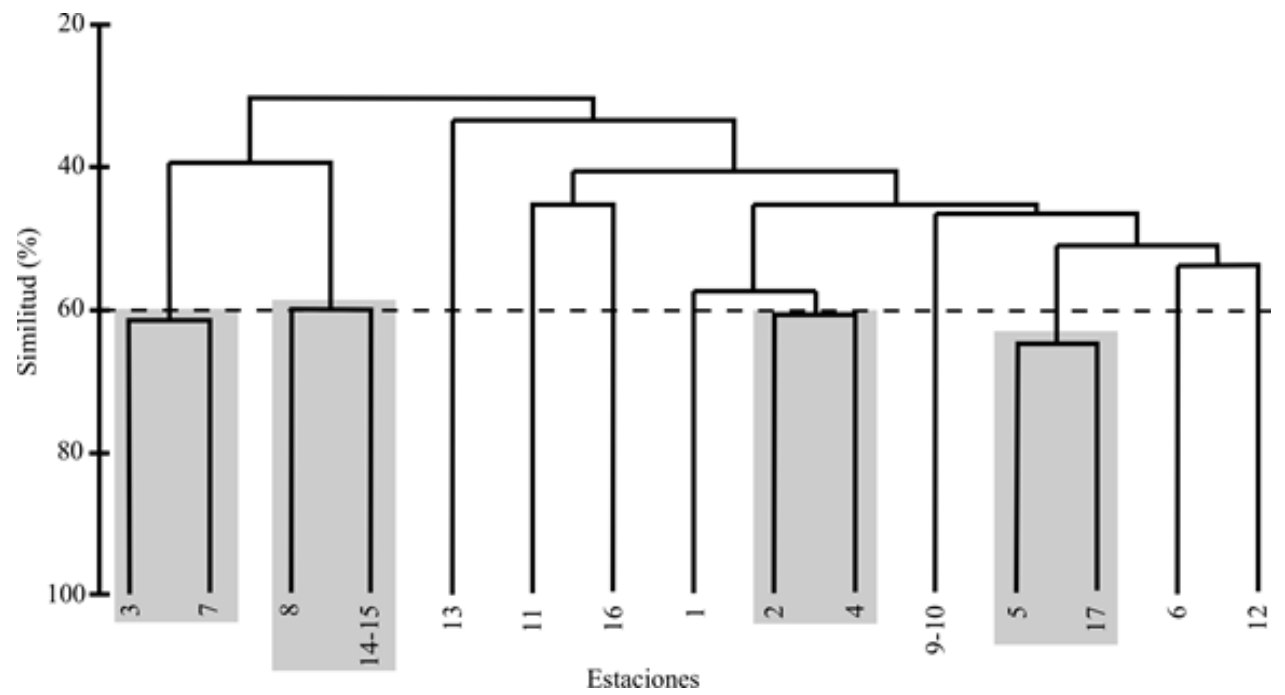

Fig.3. Dendrograma que muestra los valores de similitud entre las estaciones muestreadas durante el programa CIMAR fiordos 16.

una estación de colecta (Ver Tabla 2). La especie Ulva prolifera constituye una nueva referencia para la región de Magallanes. Acrosiphonia pacífica y Ulotrix flacca corresponden a especies presentes tanto en Magallanes como en el continente antárctico (Ver tabla 2).

De la división Ochrophyta (Clase Phaeophyceae) las especies con mayor ocurrencia fueron Macrocystis pyrifera (Linnaeus) C. Agardh presente en 14 de las 17 estaciones de colecta y Adenocystis utricularis (Bory de Saint-Vincent) Skottsberg y Caepidium antarcticum Agardh presente en 13 de 17 estaciones (Ver Tabla 2). Las especies con menos ocurrencia fueron Chordaria magellanica Kylin, Cladostephus spongiosus (Hudson) C. Agardh, Desmarestia chordalis J.D. Hooker \& Harvey, Desmarestia muellerii M. E. Ramírez \& A. F. Peters y Ectocarpus siliculosus (Dillwyn) Lyngbye, todas presentes en al menos una estación de colecta. Registrándose Se registran como nuevas referencias para la región de Magallanes a las especies Colpomenia sinuosa (Mertens ex Roth) Derbés \& Solier in Castagne, Dictyota dichotoma (Hudson) J.V. Lamouroux y Lessonia sp (especie en proceso de publicación). Dentro de esta división las especies presentes tanto en la región de Magallanes como en el continente antárctico son Halopteris obovata (J.D. Hooker \& Harvey) Sauvageau, Scytosiphon lomentaria
(Lyngbye) y Scytothamnus fasciculatus (J.D. Hooker \& Harvey) A.D. Cotton (Ver Tabla 2).

Las especies más frecuentes de la división Rhodophyta fueron Lophurella hookeriana (J.Agardh) Falkenberg presente en 12 de 17 estaciones de colecta, seguido por Ptilonia magellanica (Montagne) J. Agardh presente en nueve estaciones de colecta (Ver Tabla 2). Mientras que Las especies menos frecuentes fueron Bostrychia scorpioides (Hudson Montagne), Callophyllis atrosanguinea (J.D. Hooker \& Harvey) Hariot, Callophyllis variegata (Bory de Saint-Vincent) Kützing, Callymenia chilensis Levring, Camontagnea oxyclada (Montagne) Pujals, Ceramium diaphanum (Lightfoot) Roth, Delesseria fueguensis Skottsberg, Heterosiphonia berkeleyi Montagne, Heterosiphonia merenia Falkenberg, Leptofauchea chiloensis (Dalen \& G.W. Saunders), Rhodymenia subantarctica R.W. Ricker y Polysiphonia morrowii Harvey.

Como nuevas referencias para la región de Magallanes se identificaron las especies Callymenia chilensis Levring, $H$. merenia y $L$. chiloensis. Dentro de esta división, las especies que se distribuyen tanto en el continente antártico como en la región de Magallanes son Ahnfeltia plicata (Hudson) E.M. Fries Ballia callitricha (C. Agardh) Kützing, C. atrosanguinea, Gigartina skottsbergii Setchell \& N.L. Gardner, Griffithsia 
antarctica Hooker f. \& Harvey e Iridaea cordata (Turner) Bory de Saint-Vincent. (Ver Tabla 2).

El dendrograma de similitud (Fig. 3), definió cuatro pares de grupos de estaciones de colecta significativos ( $>60 \%$ Similitud), el primero agrupa a las estaciones 3 (Seno Otway-Entrada) y 7 (Seno Agostini), el segundo par a las estaciones 8 y 1415 (Bahía Yendegaia), el tercero correspondiente a la estación 2 (Isla Riesco) y estación 4 (Estrecho de Magallanes- sector Tortuoso), y el último par a la estación 5 (Estrecho de Magallanes- Cabo Holland) y estación 17 (Bahía Douglas) (Fig. 3).

\section{DISCUSIÓN Y CONCLUSIONES}

De las 57 especies identificadas, 50 se encuentran en la lista de especies previamente registradas para la Región de Magallanes y Tierra del Fuego (Ramírez, 2010), las 7 especies restantes son nuevas referencias y correspnden a: Ulva prolifera (Chlorophyta), Colpomenia sinuosa, Dictyota dichotoma, y Lessonia sp. (en proceso de publicación) (Ochrophyta; Clase Phaeophyceae), Callymenia chilensis, Heterosiphonia merenia y Leptofauchea chiloensis (Rhodophyta). De las especies de macroalgas identificadas en este trabajo, 11 de ellas co-ocurren también en la Antártica (Ramírez, 2010).

De las especies pertenecientes a la división Chlorophyta, C. fragile comparte el hábitat con Porphyra sp, en algunos sectores que se caracterizan por presentar un sustrato rocoso inclinado, principalmente en sectores expuestos a condiciones oceánicas y salinidades de 32 psu. (Soto et al. 2012). En este trabajo, esta alga fue colectada en sectores semi-expuestos y protegidos al oleaje, siempre asociados a salinidades de 27,7 psu. Cabe señalar, que especies del género Codium, en particular C. fragile, ha sido referida como invasora, debido a su capacidad de colonizar diversos hábitats (Williams et al . 2007), sin embargo, en la región de Magallanes, ocurre en ambientes prístinos con baja intervención antrópica. Otra especie frecuente perteneciente a esta división es A. pacifica, especie que ocurre en sectores tanto expuestos como protegidos de salinidad entre 20 a 32 psu, principalmente al interior de fiordos en el sector norte de la región de Magallanes (Soto et al. 2012). Su alta frecuencia se puede asociar a la gran plasticidad fenotípica del género, que le permite tener diferencias morfológicas de carácter adaptativo (Sussmann, 2011).

Una de las especies más conspícua es M. pyrifera (Ochrophyta-Clase Phaeophyceae) presente en gran parte de fiordos y canales de la región, información coincidente con otras investigaciones realizadas (Dayton, 1974; Searles et al. 1974; Ojeda \& Santelices, 1984; Plana et al. 2007; Ríos et al. 2007; Mansilla \& Avila, 2011). Esta especie se distribuye principalmente en sectores poco expuestos y en sustratos rocosos o de bloques y cantos característicos de esta zona (Santelices, 1989). Sus características morfológicas la convierten en una especie ingeniera, importante en la región de Magallanes, ya que proporciona alimentación, refugio, lugar de reproducción y hábitat a muchas especies tanto de algas como otros organismos marinos vertebrados e invertebrados (Adami \& Gordillo, 1999; Viddi \& Lescrauwaet, 2005; Vanella et al. 2007). Otra especie representativa para esta división es A. utricularis, la cual por lo general habita lugares protegidos o semi-expuestos con baja salinidad (Quartino et al. 2005). Es una especie que crece asociada a otras especies de macroalgas como L. chiloensis, L. hookeriana y Ulva sp. En este estudio se observó a A. utricularis, en el submareal de norte a sur en los fiordos y canales de Magallanes, en sectores con influencia tanto de glaciares como de aguas del Océano Pacífico y Atlántico.

Por último, las especies más representativas de la división Rhodophyta fueron L. hookeriana, generalmente presente en sectores poco expuestos como canales y bahías, también en sustratos rocosos (Skottsberg, 1921a; Mendoza \& Nizovoy, 2000; Boraso et al. 2003) y P. magellanica, presente en sustratos arenosos y rocosos, tanto en el intermareal bajo como en el submareal (Skottsberg, 1923; Mendoza \& Nizovoy, 2000).

De las estaciones que presentaron un alto porcentaje de similitud, se puede establecer que el grupo formado por la estación 3 (Seno OtwayEntrada) y la estación 7 (Seno Agostini), son sectores que comparten las características de ser zonas expuestas, con baja salinidad, una elevada pluviosidad y presencia de glaciares, (Valdenegro \& Silva, 2003). Este hábitat es propicio para las especies comunes encontradas en estos sectores, 
tales como: G. skottsbergii, que ocurre en hábitats de tipo rocoso y entre profundidades de 5 a 15 m (Ramírez \& Santelices, 1991; Romo et al., 2001; Mendoza \& Nizovoy, 2000). En el grupo formado por las estaciones 8 (Entrada de Canal Parry-Marinelli) y 14-15 (Bahía Yendehaia), existe presencia de $M$. pyrifera, y la salinidad promedio es mayor a 30 psu, encontrándose además las especies $A$. utricularis y $S$. lomentaria. (Skottsberg, 1921b; Hoffmann \& Santelices, 1997; Soto et al. 2012). En el caso de la especie A. utricularis también habita en zonas con salinidades de 31 psu (Soto et al. 2012). El grupo formado por la estación 2 (Isla Riesco) y estación 4 (Estrecho de Magallanes- sector Canal Tortuoso), corresponden a estaciones con distintos grados de exposición, ambas presentan influencia de aguas provenientes del Océano Pacifico con salinidades promedio de $30 \mathrm{psu}$, permitiendo la ocurrencia de C. fragile (González \& Santelices, 2004). Según Lüning (1984) la tolerancia a la temperatura en C. fragile en el hemisferio norte va desde los $0^{\circ} \mathrm{C}$ hasta los $30^{\circ} \mathrm{C}$, demostrando su amplia tolerancia ambiental y su capacidad adaptativa. En este grupo coinciden también especies como A. utricularis, Acanthococcus antarcticus Hooker \& Harvey, $B$. callitricha y $P$. magellanica, características tanto del intermareal como del submareal somero, además de G. skottsbergii y L. hookeriana presentes en ambos sectores, creciendo sobre sustratos rocosos y arenosos (Goff et al. 1992; Silva, 1992; Hoffmann \& Santelices, 1997; Mendoza \& Nizovoy, 2000; Soto et al. 2012). Por último, el grupo constituido por la estación 5 (Estrecho de Magallanes- Cabo Holland) y estación 17 (Bahía Douglas), ambos sectores son expuestos en el sistema de fiordos y canales, con una salinidad promedio de 30 psu. Estas estaciones comparten la presencia de las siguientes especies: A. utricularis, Caepidium antarcticum J. Agardh, especie que habitan sectores con salinidades $>30$ psu con límite de $\geq 32$ psu (Soto et al. 2012). Otras especies que co-ocurren en este grupo son las especies del género Lessonia, características del submareal rocoso de la región de Magallanes (Mendoza \& Nizovoy, 2000). Aun cuando se discute que la temperatura óptima de crecimiento de estas especies de Laminariales oscila entre los $10^{\circ} \mathrm{C}$ y $15^{\circ} \mathrm{C}$ (Bolton \& Lüning, 1982; Ávila et al. 1985;
Dieck, 1993), en estas latitudes se desarrollan favorablemente a temperaturas inferiores.

El sistema de fiordos y canales de Magallanes presenta en general salinidades promedio de $31,4 \pm 0,9$ psu, temperaturas promedio de $6,9 \pm 0,7$ ${ }^{\circ} \mathrm{C}$, aguas con concentraciones promedio de oxígeno disuelto de $6,6 \pm 0,2 \mathrm{ml} / \mathrm{l}^{-1}$, concentraciones de fosfato promedio de $1 \pm 0,05 \mu \mathrm{M}$ y de nitrato promedio de 8,4 $\pm 1,1 \mu \mathrm{M}$ (Valdenegro \& Silva, 2003). El aspecto climatológico de la región establece una situación bioecológica especial, debido a fenómenos que determinan existencias de sectores más lluviosos en la ribera occidental y secos en la ribera oriental, consecuencia de la acción del anticiclón del Pacifico sur sobre el borde oriental, también la topografía de la zona, juega un papel importante presentando hacia el Pacifico una serie de ventisqueros y corrientes oceánicas (Alveal et al. 1973).

Dadolos resultadosobtenidos en este trabajo se puede concluir que la dinámica de los parámetros físicos presentes en cada estación de muestreo, influye de manera directa en la composición de la flora marina bentónica. Es así como un sector con presencia de glaciares no presenta la misma composición florística que sectores con ausencia de éstos, ya que el avance y retroceso del hielo causa efectos sobre los parámetros físico-químicos como transparencia e iluminación, salinidad, nutrientes y consecuentemente en las comunidades bentónicas, que interfieren en la columna de agua y en el fondo marino (Ríos, 2007)2. No obstante, algunas especies tienen amplia tolerancia y pueden ocurrir en hábitat muy diferentes siendo posible encontrar diversidad florística similar en sectores con distintos tipos de sustrato, presencia o ausencia de bosques de $M$. pyrifera, de glaciares, aportes de sedimento o agua dulce provenientes de ríos, grado de exposición al oleaje $\mathrm{y}$ de velocidades y direcciones de corrientes. De las 57 especies identificadas en este estudio, 11 especies son comunes entre la región de Magallanes y el Continente Antártico, corroborándose la existencia de una relación florística entre ambos continentes como mencionado por Wienke \& Clayton (2002).

\section{AGRADECIMIENTOS}

Los autores agradecen a la tripulación del B/O AGOR Vidal Gormaz de la Armada de 
Chile por el apoyo recibido durante la realización del Crucero CIMAR FIORDOS 16. La presente investigación fue realizada en el marco del Proyecto "Macroalgas Marinas Bentónicas de la Región de Magallanes y Antártica Chilena" financiada por el Comité Oceanográfico Nacional. A la Iniciativa Científica Milenio (P05-002 ICM, Chile) y Programa de Financiamiento Basal- Conicyt (Nº PFB-23, Chile). Los autores agradecen a la Dra. Juliet Brodie - Natural History Museum, UK y al Dr. S.M. Boo - Chumgnam University of Korea, por su apoyo taxonómico. S. Rosenfeld agradece al programa de Magíster en Ciencias con Mención en Manejo y Conservación de Recursos Subantárticos de la Universidad de Magallanes y a la beca de Magíster del Proyecto ICM, código P05-002 otorgada por el Instituto de Ecología y Biodiversidad.

\section{LITERATURA CITADA}

Adami, M. L. \& S. Gordillo 1999. Structure and dynamics of the biota associated with Macro-cystis pyrifera (Phaeophyta) from the Beagle Channel, Tierra del Fuego. Scientia Marina, 63(1): 183-191.

Adams, N. M. (1994). "Seaweeds of new Zealand", an illustrated guide.Canterbury University Press in Christchurch, N. Z. 360 pp.

Alveal, K., H. Romo, \& J. Valenzuela (1973). Consideraciones ecológicas de las regiones de Valparaíso y Magallanes. Revista de Biología Marina, 15: 1-29.

Alveal, K. Romo \& H. Ávila M. (1982). Estudios del ciclo de vida de Macrocystis pyrifera de Isla Navarino, Chile. Gayana 39: 1-12.

Ávila, M., K. Alveal \& H. Romo (1982). Comunidades de algas epifitas en Macrocystis pyrifera de isla Navarino, Chile. Gayana Botánica, 38: 1-16.

Arntz, W., S. Thatje, D. Gerdes, J. M. Gili, J. Gutt, U. Jacob, A. Montiel, C. Orejas \& N. Teixidó. (2005). The Antarctic-Magellan connection: macrobenthos ecology on the shelf and upper slope, a progress report. Scientia Marina, 69: 237-269.

Barker, P. F. (2001). Scotia Sea regional tectonic evolution: implications for mantle flow and palaeocirculation. Earth-Science Reviews, 55(1): 1-39.

Bischoff-Bäsmann, B. \& C. Wiencke (1996). Temperature requirements for growth and survival of Antarctic Rhodophyta. Journal of Phycology, 32: 525-535.

Bolton, J. J. \& K. Lüning (1982). Optimal growth and maximal survival temperatures of Atlantic Laminaria species (Phaeophyta) in culture. Marine Biology, 66(1), 89-94.

Boraso, A. L., A. E. Rico, S. Perales, L. Pérez \& H. Zalazar (2003). Algas marinas de la Patagonia. Una guía ilustrada. Asociación de Cs. Naturales Félix de Azara. Buenos Aires, Argentina. 54 pp.

Bray, R. J \& J. I. Curtis (1957). An ordination of the upland forest communities of southern Wisconsin. Ecological Monographs, 27: 325-349.

Breeman, A. M. (1988). Relative importance of temperature and other factors in determining geographic boundaries of seaweeds: experimental and phenological evidence. Helgoländer Meeresuntersuchungen, 42: 199-241.

Brodie, J. C., A. Maggs \& D. M. John (2007). Green Seaweeds of Britain and Ireland, British Phycological Society, London, 250 pp.

Buschmann, A. H, D. Varela, M. Cifuentes, M. C. Hernández-González, L. Henríquez \& R. Westermeier (2004). Experimental indoor cultivation of the carrageenophytic red alga Gigartina skottsbergii. Aquaculture, 241(1-4), 357-370.

Buschmann, A. H., J. Correa, R. Westermeier, M. Paredes, D. Aedo, P. Potin, G. Aroca, J. Beltrán \& M. Hernandez (2001). Cultivation of Gigartina Skottsbergii (Gigartinales, Rhodophyta): Recent advances and challenges for future. Journal of Applied Phycology. 13: 255-266.

Camus, P.A. (2001). Biogeografía Marina de Chile continental. Revista Chilena de Historia Natural, 74: 587-617.

Clarke, K. R. \& R. N. Gorley (2006). PRIMER v6: User manual/Tutorial. PRIMER-E Ltd. Plymouth Marine Laboratory, U. K. 190 pp. Clarke, K. R. \& R. Gorley (2005). Primer-E 
version 6.0. Plymouth, UK Natural environmental research council, Plymouth marine laboratory, $91 \mathrm{pp}$.

Clarke, K. R., P. J. Somerfield \& R. N. Gorley (2008). Exploratory null hypothesis testing for community data: similarity profiles and biota-environment linkage. Journal of Experimental Marine Biology and Ecology, 366: 56-69.

Cormaci. M., G. Furnari \& B. Scammacca (1992). The benthic algal flora of Terra Nova Bay (Ross Sea, Antarctica). Botanica Marina, 35:541-552.

Dayton, P. K. (1974). Kelp communities of southern South America. Antarctic Journal of the U.S. IX (1): 22.

Dayton, P. K. (1985). The structure and regulation of some South American kelp communities. Ecological Monographs, 447-468.

Eggert, A. (2012). Seaweed Responses to Temperature Seaweed Biology. In C. Wiencke \& K. Bischof (Eds.), 219: 47-66. Springer Berlin Heidelberg.

Etcheverry, H. (1986). Algas bentónicas, biotopo Montemar (Chile), 22 (2): 169-226.

Fernández, M., E. Jaramillo, P. A. Marquet, C. A. Moreno, S. A. Navarrete, F. P Ojeda, C. R. Valdovinos \& J. A. Vásquez (2000). Diversity, dynamics and biogeography of Chilean benthic nearshore ecosystems: an overview and guidelines for conservation. Revista Chilena de Historia Natural, 73: 797-830.

Fredericq, S. \& M. E. Ramírez (1996). Systematic studies of the Antarctic species of the Phyllophoraceae (Gigartinales, Rhodophyta) based on $\mathrm{rbcL}$ sequence analysis. Hydrobiologia, 326/327:137-143.

Gerdes, D. \& A. Montiel (1999). Distribution patterns of macrozoobenthos: a comparison between the Magellan region and the Weddell Sea (Antarctic). Scientia Marina, 63(Suppl. 1): 149-154.

Goff, L. J., L. Liddle, P. C. Silva, M. Voytek \& A. W. Coleman (1992). Tracing species invasion in Codium, a siphonous algae, using molecular tools. American Journal of Botany, 79: 1279-1285.

González A. \& B. Santelices (2004). A dichotomous species of Codium (Bryopsidales, Chlorophyta) is invading northern Chile. Revista Chilena de Historia Natural 77: 293-304.

González-Wevar, C., T. Nakano, J. I. Cañete \& E. Poulin (2010). Molecular phylogeny and historical biogeography of Nacella (Patellogastropoda: Nacellidae) in the Southern Ocean. Molecular Phylogenetics and Evolution, 56: 115-124.

Gutt, J., E. Helsen, W. Arntz \& A. Buschmann (1999). Biodiversity and community structure of the mega-epibenthos in the Magellan region (South America). Scientia Marina, 63 (Suppl. 1): 155-170.

Hariot, P. (1889). Algues. Mission Scientifique du Cape Horn, 1882-1883. Botanique 5:1109.

Herráiz, S. C. (2009). "Consecuencias geográficas del cambio climático”. En: Cambio climático, ¿un desafío a nuestro alcance?. Ramos Castellanos (Ed.) Salamanca, Universidad de Salamanca, ISBN 978-84-7800-273-3, págs. 47-81

Hoffmann, A. \& B. Santelices (1997). Flora Marina De Chile Central. Ediciones Universidad Católica de Chile, Chile, 434pp. John, D., I. Tittley, W. Lawson \& P. Pugh (1994b). Distribution of seaweeds floras in the Southern Oceans. Botanica Marina, 37:235-239.

John, D., P. Pugh \& I. Tittley (1994a). Observations on the benthic marine algal flora of South Georgia: a floristic and ecological analysis. Bulletin of the British Museum of Natural History, 24(2): 101114.

Kristiansen, A. A., P. M. Pedersen \& L. Moseholm (1994). Salinity-temperature effects on growth and reproduction of Scytosiphon lomentaria (Fucophyceae) along the salinity gradient in Danish waters. Phycologia, 33(6), 444-454.

Kylin, H. \& C. Skottsberg (1919). Zur kenntnis der Subantarktischen und Antarktischen Meeresalgen II. Rhodophyceen. In Nordensjold, O. (Ed). Wissenschaftliche Ergebnisse der Swedischen Sudpolar Expedition 1901-1903 4: 2(15):1-88. 
Lamb, I. \& M. Zimmermann (1977). Benthic marine algae of the Antarctic Peninsula. Antarctic Res. Ser. Washington 23:129229.

Lemoine, P. (1920). Botanische Ergebnisse der Swedischen Expedition nach Patagonien und der Feurlande 1907-1909. VII. Les Melobesiees. Kongliga Svenska Vetenskaps Akademiens Handlingar, 61 (4):1-17.

Levring, T. (1960). Contribution to the marine algal flora of Chile. Acta Universitatis Lundensis 56(10): 1-85.

Lüning, K. (1984). Temperature tolerance and biogeography of seaweeds: the marine algal flora of Helgoland (North Sea) as an example. Helgoländer Meeresuntersuchungen, 38(2), 305-317.

Mansilla, A. \& N. Navarro (2003). Contribución al estudio de la flora ficológica de las islas Diego Ramírez (Chile). En: Memorias Curso Internacional de Postgrado y Especialización de Macroalgas en Ambientes Subantárticos (eds. Mansilla, A., C. Werlinger \& N. Navarro). pp. 85-89.Ediciones Universidad de Magallanes, Punta Arenas.

Mansilla, A., N. Navarro \& M. Fujii (2006). First record of a ceramiaceous red algal species, Ceramium stichidiosum, from magellanic region, Chile. Gayana, 70 (2): 245-251.

Mansilla, A. \& M. Ávila (2011). Using Macrocystis pyrifera (L.) C. Agardh from southern Chile as a source of applied biological compounds. Revista Brasileira de Farmacognosia, 21(2): 262-267.

Mendoza, M. L. \& A. Nizovoy (2000). Géneros de Macroalgas marinas de la Argentina, fundamentalmente de Tierra del Fuego. Editado por el Poder Legislativo de la Provincia de Tierra del Fuego, Antártida e Islas del Atlántico Sur. Argentina. 142 p.

Meneses, I. \& B. Santelices (2000). Patterns and breaking points in the distribution of benthic algae along the temperate Pacific coast of South America. Revista chilena de historia natural, 73(4), 615-623.

Michelou, V. K., J. G. Caporaso, R. Knight \& S. R. Palumbi (2013). The Ecology of Microbial Communities Associated with Macrocystis pyrifera. Plos One, 8(6), e67480.
Moe, R. \& P. Silva (1989). Desmarestia Antarctica (Desmarestiales, Phaeophyceae) A newligulate species with an endophytic gametophyte. Plant Systematics and Evolution, 164: 273-283.

Moe, R. \& P. Silva (1979). Morphological and taxonomic studies on Antarctic Ceramiaceae (Rhodophyceae). I. Antarcticothamnion polysporum gen. ET sp. Nov. British Phycological Journal, 14:385-405.

Moe, R. \& P. Silva (1980). Morphological and taxonomic studies on Antarctic Ceramiaceae (Rhodophyceae). II. Pterothamnion antarcticum (Kylin) comb. nov. (Antithamnion antarcticum Kylin). British Phycological Journal, 15: 1-17.

Moe, R. \& P. Silva (1983). Morphological and taxonomic studies on Antarctic Ceramiaceae (Rhodophyceae) III. Georgiella and Pluma-riopsis. (Tribe Ptiloteae). British Phycological Journal, 18:275-298.

Moe, R. (1985). Gainia and Gainiaceae, a new genus and family of crustose marine Rhodophyceae from Antarctica. Phycology, 24:419-428.

Moe, R. (1986). Notophycus fimbriatus (Solieraceae), a new genus and species of marine Rhodophyceae from the Antarctic Peninsula. Phycologia, 25: 544-550.

Moreno, C. A. \& F. H. Jara (1984). Ecological studies on fish fauna associated with Macrocystis pyrifera belts in the south of Fuegian Islands, Chile. Marine Ecology Progress Series, 15: 99-107.

Ojeda, F. P. \& B. Santelices (1984). Invertebrate communities in holdfasts of the kelp Macrocystis pyrifera from southern Chile. Marine Ecology Progress Series, 16: 6573.

Plana, J., A. Mansilla, M. Palacios \& N. Navarro (2007). Estudio poblacional de Macrocystis pyrifera (L.) C. Agardh (Laminariales: Phaeophyta) en ambientes protegidos $y$ expuestos al oleaje en Tierra del Fuego. Gayana, 71(1): 65-77.

Pérez, V. (2000). Cronología de antecedentes para la Historia de las Ciencias Naturales de la Región de Magallanes: Siglo XVI al XIX. Ediciones de la Universidad de 
Magallanes, Punta Arenas, Chile. 235 pp.

Quartino, M. L., H. E. Zaixso \& A. L. Boraso de Zaixso (2005). Biological and environmental characterization of marine macroalgal assemblages in Potter Cove, South Shetland Islands, Antarctica. Botánica Marina, 48(3), 187-197.

Ramírez, M. E. \& B. Santelices (1991). Catálogo de las algas marinas bentónicas de la costa temperada de Pacifico de Sudamérica, Facultad de Ciencias Biológicas Pontificia Universidad Católica de Chile, Chile, 437pp.

Ramírez, M. E. (1995). Recolección y colecciones científicas de macroalgas marinas. En E. K. Alveal, Manual de Metodos Ficologicos (págs. 417-428). Concepción, Chile: Universidad de Concepción.

Ramírez, M. E. (2010). Flora marina Bentónica de la región austral de Sudamérica y la Antártica. Anales del Instituto de la Patagonia (Chile), 38(1):57-71.

Ricker, R. (1987). Taxonomy and Biogeography of Macquarie Islands Seaweeds. British Museum Natural History. London, 344 pp.

Ríos, C., E. Mutschke \& A. Montiel (2010). Estructura de la comunidad macrofaunística bentónica en la boca oriental del estrecho de Magallanes, Chile austral. Anales del Instituto de la Patagonia, 38(1): 83.96

Ríos, C. \& E. Mutschke (2009). Aporte al conocimiento de Macrocystis pyrifera: revisión bibliográfica sobre los "huirales" distribuidos en la región de Magallanes. Anales del instituto de la Patagonia, 37(1): 97-102

Ríos, C., W.E. Arntz, D. Gerdes, E. Mutschke \& A. Montiel (2007). Spatial and temporal variability of the benthic assemblages associated to the holdfasts of the kelp Macrocystis pyrifera in the Straits of Magellan, Chile. Polar Biology, 31:89100.

Romo, H., K. Alveal \& M. Ávila (1984). Efectos de la poda en la sobrevivencia, tamaño y rendimiento de Macrocystis pyrifera (L. Ag.) (Lessoniaceae) de Isla Navarino, Chile. Gayana Botánica, 41(1-2): 127-135.

Romo, J., M. Ávila \& A. Candia (2001). Manual de técnicas de cultivo y repoblación de "luga roja" (Gigartina skottsbergii). Proyecto FONDEF D97I1064. IFOPUniversidad de Concepción, Chile. pp. 32.

Rozzi, R., F. Massardo, A. Berghöfer, C. B. Anderson \& A. Mansilla (2006). La Reserva de Biosfera Cabo de Hornos. Programa MaB-UNESCO. Ediciones Universidad de Magallanes, Punta Arenas.

Rozzi, R., F. Massardo, A. Mansilla, C. B. Anderson \& A. Berghöfer (2007). La Reserva de Biosfera Cabo de Hornos: Un desafío para la conservación de la biodiversidad e implementación del desarrollo sustentable en el extremo austral de América. Anales del Instituto de la Patagonia, Ciencias Naturales, 35: 55-62.

Rozzi, R., J. J. Armesto, J. Gutiérrez, F. Massardo, G. Likens, C. B. Anderson, A. Poole, K. Moses, G. Hargrove, A. Mansilla, J. H. Kennedy, M. Willson, K. Jax, C. Jones, J. B. Callicott \& M. T. Kalin (2012). Integrating ecology and environmental ethics: Earth stewardship in the southern end of the Americas. BioScience, 62 (3): 226-236.

Santelices, B. (1989). Algas marinas de Chile. Distribución. Ecología. Utilización. Diversidad. Ediciones Universidad Católica de Chile. Santiago.

Searles, R. B., G. L. Leister \& J. F. Brauner (1974). Seaweds of southern South América. Antarctic Journal of the U.S. IX (1): 23-24.

Silva, P. (1992). Geographic patterns of diversity in benthic marine algae. Pacific Science, 46: 429-437.

Skottsberg, C. (1907). Zur kenntnis der Subantarktischen und Antarktischen meeresalgen I. Phaeophyceen. In: Nordensjold O. (Ed). Wissenschaftliche Ergebnisse der Schwedischen Sudpolar Expedition 1901-1903. 4 (6): 1-172.

Skottsberg, C. (1921a). Botanische Ergebnisse der schwedischen Expedition nach Patagonien und der Feuerland 1907-1909, IX. Marine Algae. 2. Rhodophyceae. Kgl. Svensk. Vet. Akad. Handl. 63 (8): 1-70.

Skottsberg, C. (1921b). Botanische Ergebnisse der schwedischen Expedition nach 
Patagonien und der Feuerland 1907-1909, VIII. Marine Algae. 1. Phaeophyceae. Kgl. Svensk. Vet. Akad. Handl. 61 (11): 1-56.

Skottsberg, G. (1923). Botanische Ergebnisse der schwedischen Expedition nach Patagonien und dem Feuerlande 1907-1909. IX. Marine Algae 2. Rhodophyceae. Kgl. Svensk. Vet. Akad. Handl. 63 (8): 1-70.

Sneath, P. H. A. \& R. R. Sokal (1973). Numerical taxonomy. The principles and practice of numerical classification. W. H. Freeman, San Franscisco. 573 pp.

Soto, E. H., P. Báez, M. E. Ramírez, S. Letelier, J. Naretto \& A. Rebolledo (2012). Biotopos Marinos intermareales entre Canal Trinidad y Canal Smyth, Sur de Chile. Revista de Biología Marina y Oceanografía, 47(2): 177-191.

Soto, M. J. (1991). Aportación al estudio sobre la fenología reproductora de las algas pardas y verdes del litoral del sureste de España. Acta Botánica Malacitana, 16 (2): 317-323.

Sussmann, A.V. \& R. A. Scrosati (2011). Morphological Variation in Acrosiphonia arcta (Codiolales, Chlorophyta) from Environmentally Different Habitats in Nova Scotia, Canada. Rhodora, 113(953), 87105.

Tom Dieck, I. (1993). Temperature tolerance and survival in darkness of kelp gametophytes (Laminariales, Phaeophyta): ecological and biogeographical implications. Marine Ecology Progress Series, 100: 253-253.

Valdenegro, A. \& N. Silva (2003). Caracterización oceanográfica física y química de la zona de canales y fiordos australes de Chile entre el estrecho de Magallanes y cabo de Hornos (CIMAR 3 Fiordos). Ciencia y Tecnología del Mar, 26(2): 19-60.

Van den Hoek, C. (1982a). The distribution of benthic marine algae in relation to the temperature regulation of their life histories. Biological journal of the Linnean Society, 18(2), 81-144.

Van den Hoek, C. (1982b). Phytogeographic distribution groups of benthic marine algae in the North Atlantic Ocean. A review of experimental evidence from life history studies. Helgoländer Meeresuntersuchungen, 35(2): 153-214.

Vanella, F.A., D. A. Fernández, M. C. Romero \& J. Calvo (2007). Changes in the fish fauna associated with a sub-Antarctic Macrocystis pyrifera kelp forest in response to canopy removal. Polar Biology 30: 449-457

Viddi, F.A. \& A. K. Lescrauwaet (2005). Insights on habitat selection and behavioural patterns of Peale's dolphins (Lagenorhynchus australis) in the Strait of Magellan. Aquatic Mammals 31: 176-183

Wiencke, C. \& M.N. Clayton (2002). Antarctic seaweeds. In: ( J. W. Wagele, Ed.). Synopsis of the antarctic benthos, A.R.G. Gantner Verlag KG. Ruggell/ Lichtensteien. 159 pp., 40 pls.

Williams, S. L. \& J. E. Smith (2007). A global review of the distribution, taxonomy, and impacts of introduced seaweeds. Annual Review of Ecology Evolution and Systematics, 38: 327-359. 